\title{
A case of west nile meningitis complicated by pontine stroke
}

\begin{abstract}
West Nile Virus (WNV) commonly causes meningitis and encephalitis. At least three cases of stroke in the setting of West Nile encephalitis (WNE) have been reported in the litrature. We report the first case of West Nile Meningitis/Encephalitis complicated by pontine stroke. A patient presenting with stroke, seizure, and meningitis should prompt a thorough workup to include viral pathogens such as WNV. Early identification and treatment of WNV meningitis/encephalitis could help prevent neuropsychiatric symptoms and decrease longterm morbidity.
\end{abstract}

Keywords: west nile virus, meningitis, vasculitis, pontine stroke
Volume 7 Issue 6 - 2017

\author{
Ikram Asad,' Berti Aldo,' Calder \\ Christopher,' Quadri Syed A, ${ }^{2}$ Zafar Atif' \\ 'Department of Neurology, University of New Mexico, USA \\ ${ }^{2}$ California Institute of Neuroscience, USA
}

Correspondence: Syed A Quadri, California Institute of Neuroscience, 21 Lynn Road, Suite 12 Thousand Oaks, CA 9136, USA, Email adnan359@gmail.com

Received:September 24, 2017 | Published: October 25, 2017

\section{Introduction}

West Nile Virus (WNV) is a common cause of viral CNS meningitis and/or encephalitis and is usually spread by mosquitoes in endemic regions. ${ }^{1}$ Rarely it may present with stroke due to vasculitis of cerebral blood vessels. ${ }^{2}$ At least three cases of stroke in the setting of WNE have been reported: a young girl with MCA stroke, elderly women with cerebellar stroke, and an elderly man with a nonspecific periventricular stroke. ${ }^{2-4}$ We report the first case of West Nile Meningitis/Encephalitis complicated by Pontine Stroke.

\section{Case summary}

A middle aged man presented with the new-onset seizures. His past medical history was significant for subarachnoid hemorrhage (SAH) due to ruptured middle carotid artery (MCA) aneurysm s/p right(R)-sided craniotomy and clipping, heart failure with reduced ejection fraction (HFrEF), Non-ST elevation myocardial infarction (NSTEMI), hypertension, diabetes, tobacco use disorder and endstage renal disease (ESRD) due to polycystic kidney disease on hemodialysis (HD). Reportedly, he was not compliant with regular dialysis and was complaining of a headache, vomiting, and fatigue a day prior to presentation. On the day of admission, seizures described as "generalized tonic-clonic" started four hours into HD. In the emergency department, the patient was notably encephalopathic and meeting SIRS criteria, which led to eventual intubation and initiation of broad-spectrum antibiotics, corticosteroids, and antiepileptic agents. Review of systems was negative and his neurologic examination was non-focal other than an extensor plantar response on the left and generalized weakness. His family reported no known allergies, denied current tobacco, alcohol, or drug use.

He underwent a lumbar puncture (LP) with cerebrospinal fluid (CSF) profile showing total white cells of 1110, Lymphocytes 55, Mononuclear 42, Glucose 27 (Serum glucose 75), Protein 193, and an opening pressure of $25 \mathrm{cmH} 20$. His CSF gram stain and culture, fungal culture, VDRL, HSV PCR were all negative as were blood cultures, TB Quantiferon gold tests, and serum cocci antibodies. The patient was admitted to the Neuroscience Intensive Care Unit for close support. CT head without contrast was negative. MRI brain showed an acute ischemic lacunar infarct in the right paramedian Pons. CTA of the head did not reveal any vascular abnormalities of anterior and posterior circulation. EEG revealed slowing without any ictal concerns. Eventually, serology came back positive for West Nile Virus IgM.

The patient was extubated on hospital day four and he completed his two-week course of antibiotics. The remainder of his hospitalization consisted of fluctuation in mental status with bouts of delirium and psychotic outbursts consisting of paranoid delusions and flight of ideas. Antipsychotic agents were given with improvement in neuropsychiatric symptoms and the patient was discharged on hospital day twenty with persistent cognitive deficits.

\section{Discussion}

As per the CDC criteria, WNV infection is confirmed by the presence of IgM on serology and pleocytosis in CSF studies. ${ }^{5}$ Seizures and stroke are not the common presentations of WNV. In a few studies, the involvement of thalamic and midbrain structures are shown in the patients who had WNV encephalitis and stroke. ${ }^{1,6,7}$ In a large study of 228 patients infected with WNV in serum it was found that prior history of stroke correlated with increased mortality, however, none of these patients suffered an acute stroke during their hospitalization. ${ }^{8}$ To our knowledge, there are only three known cases of stroke in the patients with acute WNV infection, and ours is the first report of isolated pontine stroke complicating WNV meningitis. It is theorized that vasculitis is the probable cause of ischemic events in such patients caused by the inflammation from viral infection. ${ }^{2}$ It is important to always have a high index of suspicion for stroke in any patient with meningitis and order an MRI. WNV meningitis/ encephalitis should always be considered in the differential in patients presenting with seizures and stroke living in endemic regions. ${ }^{9}$

\section{Conclusion}

This case demonstrates the importance of clinical insight into the rare causes of stroke. A patient presenting with stroke, seizure, and meningitis should prompt a thorough workup to include viral pathogens such as WNV. Early identification and treatment of WNV meningitis/encephalitis could help prevent neuropsychiatric symptoms and decrease long-term morbidity.

\section{Acknowledgements}

None. 


\section{Conflict of interest}

Authors reports no conflict of interest.

\section{Funding}

None.

\section{References}

1. Gea-Banacloche J, Johnson RT, Bagic A, et al. West Nile virus: pathogenesis and therapeutic options. Ann Intern Med. 2004;140(7):545-553

2. Alexander JJ, Lasky AS, Graf WD. Stroke associated with central nervous system vasculitis after West Nile virus infection. J Child Neurol. 2006;21(7):623-625.

3. Kulstad EB, Wichter MD. Wichter, West Nile encephalitis presenting as a stroke. Ann Emerg Med. 2003;41(2):283.
4. Whitney EA, Heilpern KL, Woods CW, et al. West Nile virus among hospitalized, febrile patients: a case for expanding diagnostic testing. Vector Borne Zoonotic Dis. 2006;6(1):42-49.

5. Petersen LR, Marfin AA. West Nile virus: a primer for the clinician. Ann Intern Med. 2002; 137(3):173-179.

6. Cerna F, Mehrad B, Luby JP, et al. St. Louis encephalitis and the substantia nigra: MR imaging evaluation. AJNR Am J Neuroradiol . 1999;20(7):1281-1283.

7. Kumar S, Misra UK, Kalita J, et al. MRI in Japanese encephalitis. Neuroradiology. 1997;39(3):180-184.

8. Bode AV, Sejvar JJ, Pape WJ, et al.West Nile virus disease: a descriptive study of 228 patients hospitalized in a 4-county region of Colorado in 2003. Clin Infect Dis. 2006;42(9):1234-1240.

9. Sejvar JJ. Clinical manifestations and outcomes of West Nile virus infection. Viruses. 2014;6(2):606-623. 